\title{
CINÉTICA DE HIDRATAÇÃO E DIFUSÃO NOS GRÃOS DE CEVADA
}

\author{
F. D. MONTANUCI ${ }^{1}$, L. M. M. JORGE ${ }^{2}$, R. M. M. JORGE ${ }^{3 *}$ \\ ${ }^{1,3}$ Universidade Federal do Paraná, Departamento de Engenharia Química, Programa de \\ Pós-graduação em Engenharia de Alimentos, Av. Cel. Francisco H dos Santos, s/n, Jardim das \\ Américas, CEP: 81530-900 - Curitiba - PR - Brasil \\ ${ }^{2}$ Universidade Estadual de Maringá, Departamento de Engenharia Química, CEP 87020-900 - \\ Maringá - PR - Brasil \\ *E-mail para contato: rjorge@ufpr.br
}

\begin{abstract}
RESUMO - A cevada é muito utilizada no processo de maltagem para produção de malte cervejeiro. O objetivo deste trabalho foi avaliar a cinética de hidratação dos grãos de cevada através do modelo de difusão. Os ensaios de hidratação foram realizados com o cultivar BRS 195, durante 32 horas nas temperaturas de 10 a $35^{\circ} \mathrm{C}$. Observou-se que a cinética de hidratação dos grãos de cevada pode ser dividia em duas fases, a primeira compreendendo as oito primeiras horas nas quais que ocorre uma rápida absorção de água e a segunda fase onde a taxa de absorção de água é menor, até a umidade entrar em equilíbrio dentro do grão. Também observou-se que o processo de hidratação é por camadas e quanto maior a temperatura maior foi a absorção de água. O coeficiente de difusão foi maior na temperatura de $20^{\circ} \mathrm{C}$ e menor nas temperaturas de 30 e $35^{\circ} \mathrm{C}$ provavelmente porque essas temperaturas são altas para o processo de hidratação ocorrendo perda de sólidos.
\end{abstract}

\section{INTRODUÇÃO}

A cevada (Hordeum vulgare sp. vulgare) é um cereal de inverno que ocupa a quinta posição mundial, em ordem de importância econômica. O grão é utilizado na industrialização de bebidas como, por exemplo, cerveja e destilados, na composição de farinhas ou flocos para panificação, na produção de medicamentos e na formulação de produtos dietéticos (Embrapa, 2014).

Amido, fibras e proteínas são os principais componentes dos grãos de cevada, que podem ser influenciados pela genética e por fatores ambientais (Oscarsson et al.,1996 e Andersson et al.,1999). A cevada é considerada um grão funcional devido à fonte de $\beta$-glucana, vitaminas do complexo B, tocotrienois e tocoferois conhecidos por reduzir o colesterol LDL através de sua ação antioxidante (Ullrich e Baik, 2008; Sharma et al., 2011). 
A maltagem dos grãos de cevada consiste em três etapas: hidratação também conhecida como maceração, germinação e secagem dos grãos. A cevada é mantida sob maceração (hidratação) em água até alcançar $44 \%$ de umidade, seguida da germinação em condições controladas de temperatura, umidade e aeração, após essa etapa os grãos são seco até cerca de $10 \%$ de umidade a temperaturas próximas de $60^{\circ} \mathrm{C}$. Na etapa de maltagem ativam-se as enzimas, que provocam modificações nas substâncias armazenadas no grão. O objetivo é produzir o mínimo de crescimento e o máximo de modificação do endosperma, produzindo grãos com alta atividade enzimática. Essas enzimas são responsáveis pela transformação do amido em açúcares, as mais importantes são $\alpha$-amilase e $\beta$ amilase (Barreiro et al., 2003).

Os modelos fenomenológicos consideram as etapas elementares de transferência de massa por difusão e/ou convecção, podem ser de parâmetros concentrados ou distribuídos e, geralmente, representam as principais tendências do processo, mesmo fora das condições experimentais em que foram validados. Os modelos de parâmetros concentrados não contemplam variações espaciais das propriedades físicas no sistema, enquanto que os de parâmetros distribuídos normalmente as representam, podendo, ambos, ser utilizados para simular o comportamento do grão durante a hidratação. Entretanto, via de regra, é difícil medir os perfis de umidade no interior dos grãos, o que dificulta a validação dos modelos de parâmetros distribuídos e assim limita a sua utilização. Neste estudo será utilizado o modelo da difusão.

O objetivo deste trabalho foi avaliar a cinética de hidratação dos grãos de cevada através do modelo de difusão. Os ensaios de hidratação foram realizados com o cultivar BRS 195, durante 32 horas nas temperaturas de 10 a $35^{\circ} \mathrm{C}$.

\section{MATERIAIS E MÉTODOS}

\subsection{Material}

Foi utilizado o Cultivar BRS 195 doado pela Embrapa Trigo localizada em Passo Fundo - RS. $\mathrm{O}$ cultivar pertence à safra de 2011 e foi cultivado na região sul do Brasil. As amostras foram armazenadas sob refrigeração a $5^{\circ} \mathrm{C}$ até a realização dos ensaios.

\subsection{Hidratação}

Os ensaios de hidratação foram realizados em banho ultratermostático (marca Solab) com temperatura controlada. Em cada ensaio foi utilizada uma massa de $250 \mathrm{~g}$ de grãos de cevada que foram colocados em beckers de $600 \mathrm{~mL}$ e submersos em água destilada.

Os experimentos foram realizados em duplicatas para cada temperatura de hidratação conforme detalhado a seguir:

- $\quad$ - As temperaturas pré-definidas para os ensaios foram: $10^{\circ}, 15^{\circ}, 20^{\circ}, 25^{\circ}$ e $35^{\circ} \mathrm{C}$; 
- - Amostras de aproximadamente 30g foram retiradas nos tempos pré-determinados. Nos ensaios as amostras de grãos foram retiradas nos seguintes instantes de tempo (horas): $0 ; 0,08 ; 0,5 ; 1 ; 1,5$; $2 ; 2,5 ; 3 ; 3,5 ; 4 ; 5,6 ; 7 ; 8 ; 10 ; 12 ; 14 ; 16 ; 18 ; 20 ; 22 ; 24 ; 26 ; 28 ; 30 ; 32$. O tempo de 32 horas é suficiente para que o processo de hidratação atinja o equilíbrio em todas as temperaturas;

- - Cada amostra de grãos foi seca superficialmente sobre papel toalha para retirar a água superficial;

- - Uma parte da amostra foi utilizada para avaliar a densidade do grão de cevada, enquanto a outra parte foi destinada à determinação da umidade.

O conteúdo de umidade das amostras de cevada foi determinado em quadruplicata pelo método da AOAC 925.10 (1995) em porcentagem de base seca.

\subsection{Modelo de difusão}

A difusão de água em uma esfera pode ser representada pela Equação 1.

$$
\frac{\mathrm{aM}}{\mathrm{dt}}=D\left(\frac{\mathrm{d}^{2} M}{\mathrm{dr}^{2}}+\frac{2}{r} \frac{\mathrm{am}}{\mathrm{dr}}\right)
$$

A solução geral da segunda lei de Fick's em coordenadas esféricas é obtida por Crank (1975) e dada pela Equação 2:

$$
M R=\frac{\left(M_{t}-M_{S}\right)}{\left(M_{0}-M_{S}\right)}=\frac{6}{\pi^{2}}+\sum_{n=1}^{\infty} \frac{1}{n^{2}} \exp \left(\frac{-D_{e} n^{2} \pi^{2}}{r^{2}} t\right)
$$

Sendo D a difusividade, $\mathrm{M}$ o conteúdo de umidade em função do tempo, $\mathrm{r}$ é a coordenada radial e t é o tempo. Uma solução analítica pode ser obtida assumindo a partícula como uma esfera, considerando volume constante durante a hidratação, coeficiente de difusão constante e independente da concentração e resistência de transporte de massa desprezível no filme. Assim obtém-se a quantidade total de água que se difunde para o interior do grão hipoteticamente esférico num dado instante. No caso de um longo tempo de hidratação, a solução apresentada na forma de somatória pode ser simplificada empregando-se apenas o primeiro termo dado pela Equação 3 com um erro menor que $0,1 \%$ (Bello et al. 2004).

$$
M R=\frac{\left(M_{t}-M_{S}\right)}{\left(M_{0}-M_{S}\right)}=\frac{6}{\pi^{2}} e^{-\left(\frac{D e \pi^{2} t}{r^{2}}\right)}
$$

$\mathrm{M}_{\mathrm{t}}$ é o conteúdo de umidade em função do tempo $(\mathrm{g} / \mathrm{g}), \mathrm{M}_{0}$ é o conteúdo de umidade inicial $(\mathrm{g} / \mathrm{g})$, $\mathrm{M}_{\mathrm{s}}$ conteúdo de umidade na saturação $(\mathrm{g} / \mathrm{g}), \mathrm{D}_{\mathrm{e}}$ é a difusividade efetiva $\left(\mathrm{m}^{2} / \mathrm{s}\right)$, t é o tempo $(\mathrm{h}), \mathrm{r}$ é a coordenada radial $(\mathrm{m})$.

A Equação 4 é obtida mediante linearização da Equação 3. 


$$
\operatorname{Ln}(M R)=-\frac{1}{2}-\frac{D_{e} \pi^{2} t}{r^{2}}
$$

A difusividade efetiva foi calculada pela Equação 4 mediante regressão linear de Ln (MR) em relação ao tempo. Geralmente a difusividade efetiva é usada devido ao limite de informações do mecanismo de movimento de umidade durante o processo de hidratação. A temperatura é um parâmetro crítico que afeta a difusão em alimentos. A dependência da difusão com a temperatura é descrita pela Equação de Arrhenius apresentada na Equação 5.

$$
D_{e}=D_{0} e^{\left(\frac{-E a}{R T_{a}}\right)}
$$

$D_{e}$ difusividade efetiva, $D_{0}$ difusividade inicial, $E_{a}$ energia de ativação, $R_{g}$ constante universal dos gases, $T a$ temperatura $(\mathrm{K})$.

\section{RESULTADOS E DISCUSSÃO}

Observa-se na Figura 1 que em todas as temperaturas analisadas a absorção de água é mais rápida nos estágios iniciais, principalmente nas primeiras oito primeiras horas e diminuiu gradualmente à medida que o conteúdo de umidade se aproxima da saturação. Segundo Kashaninejad et al, (2007) e Khazaei and Mohammadi (2009) a inclinação dos dois segmentos lineares corresponde à primeira e à segunda fases de absorção de água denominadas de fase de difusão e fase de estabilização. De forma geral o tempo de duração da primeira fase para a segunda depende de fatores tais como o conteúdo de umidade inicial, temperatura da água e do produto.

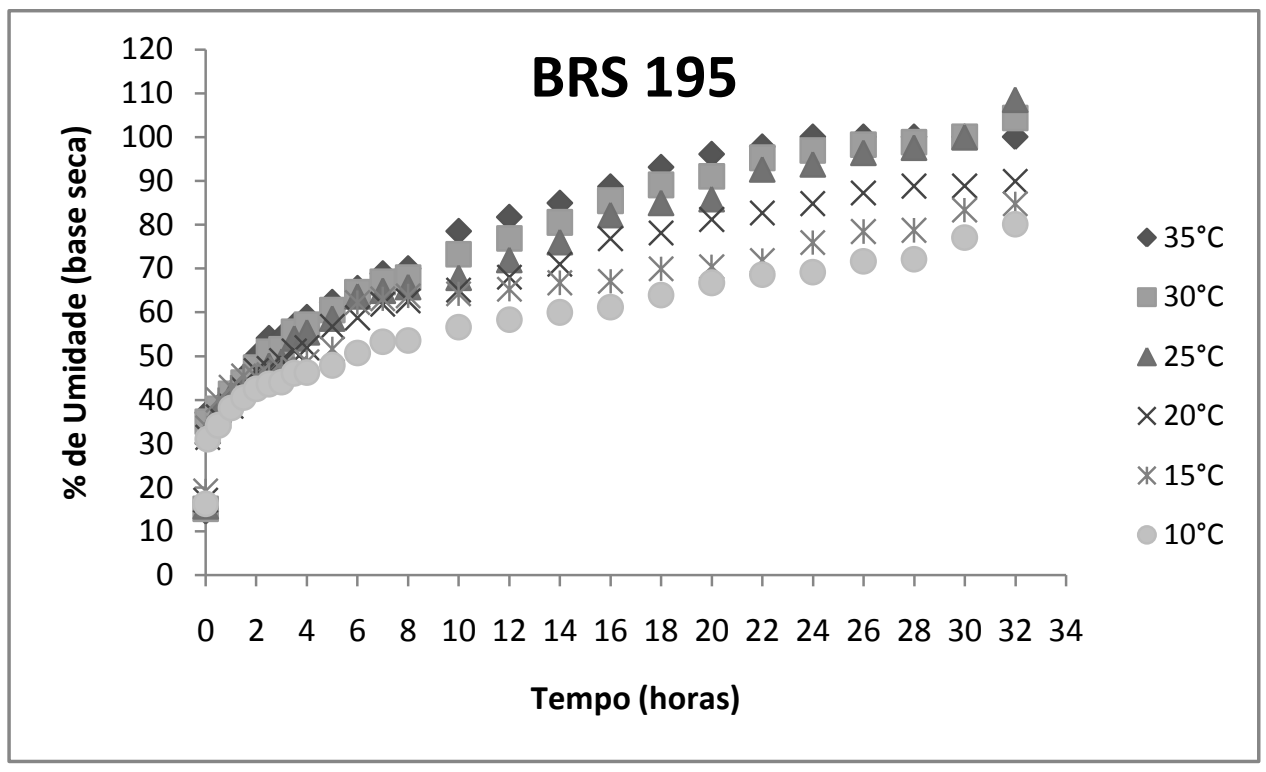

Figura 1 - Isotermas de hidratação do Cultivar BRS 195 
Também pode-se observar na Figura 2 que a hidratação da cevada é realizada em camadas. A Figura 2 é a ampliação da cinética de hidratação do cultivar BRS 195 nas temperaturas de 35 e $15^{\circ} \mathrm{C}$.

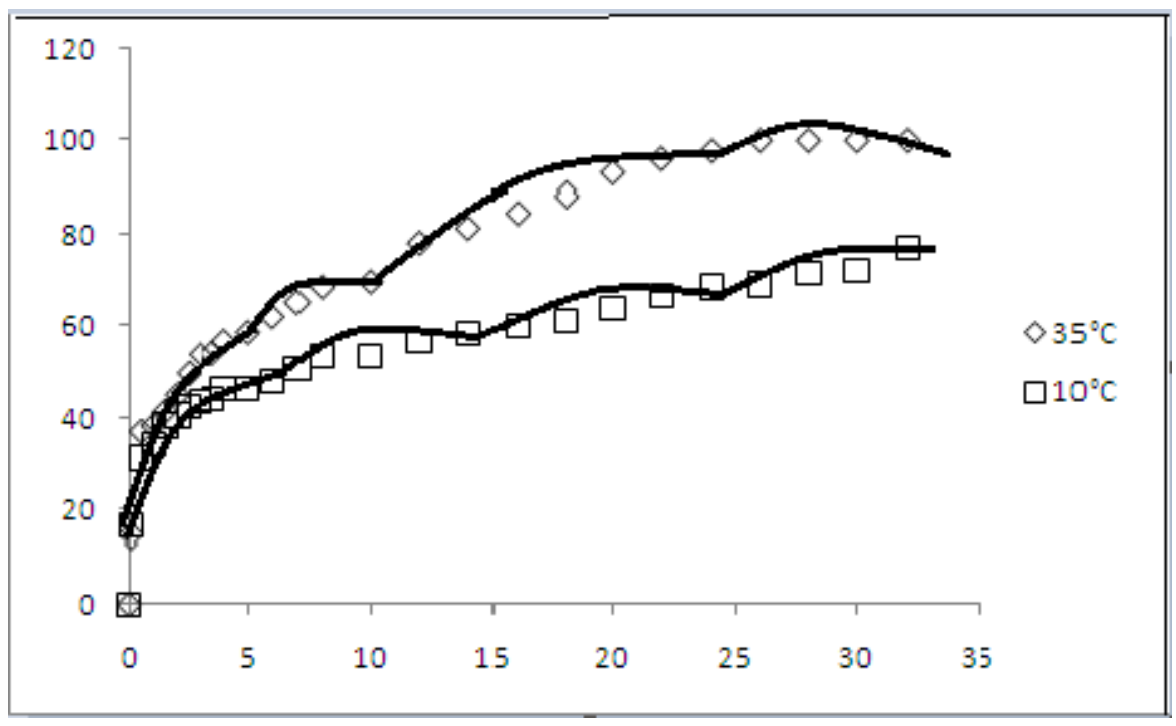

Figura 2 - Cinética de hidratação do cultivar BRS 195 nas temperaturas de 35 e $15^{\circ} \mathrm{C}$.

Observou-se que quanto maior a temperatura, maior é a velocidade de absorção de água no processo de hidratação, concordando com Geankoplis (1983) que afirma que a velocidade de hidratação aumenta com o aumento da temperatura.

A difusividade é uma propriedade de transporte importante, sendo o conhecimento desta propriedade necessário para o projeto e otimização de todos os processos que envolvem o movimento interno de umidade (Prasad et al., 2010). O gráfico de $\operatorname{Ln}\left[\left(\mathrm{X}_{\mathrm{t}}-\mathrm{X}_{\mathrm{e}}\right) /\left(\mathrm{X}_{0}-\mathrm{X}_{\mathrm{e}}\right)\right]$ versus tempo apresentado na Figura 3 gera uma reta a partir da qual pode ser calculado o valor do coeficiente de difusão efetivo. 


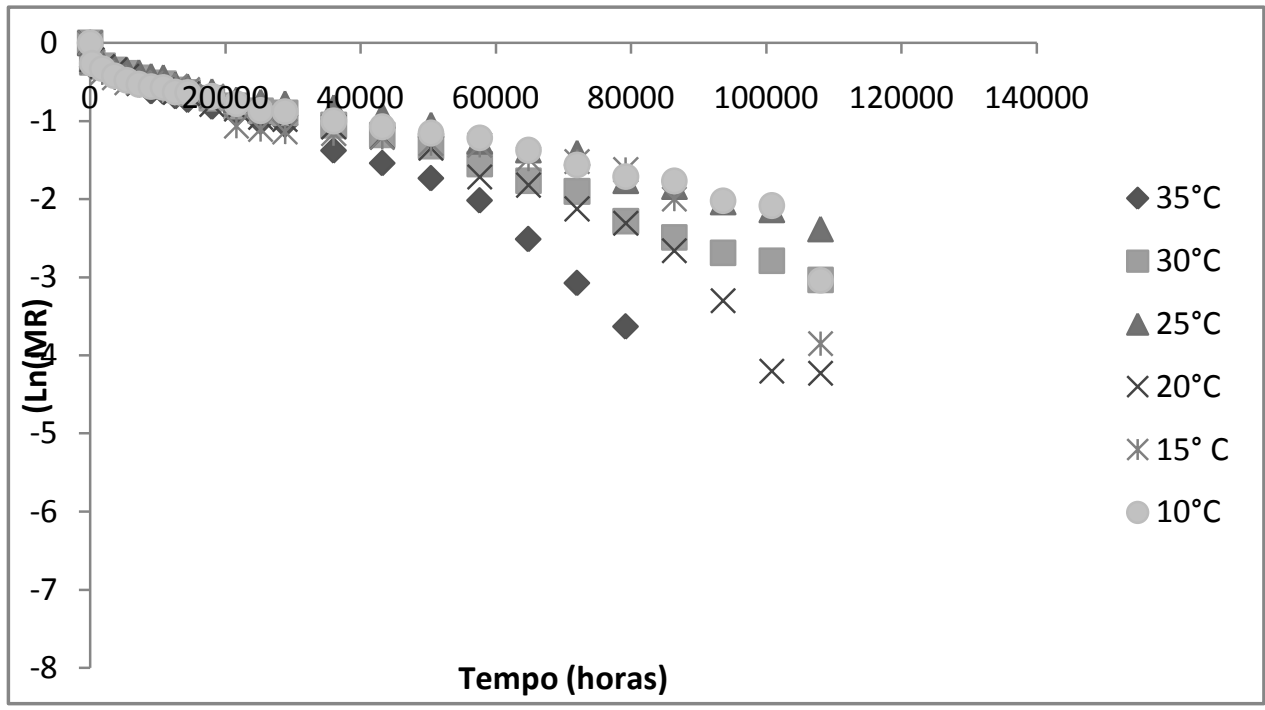

Figure 3 - Determinação do coeficiente de difusão dos grãos de cevada para o cultivar BRS 195

Os coeficientes difusão efetivos apresentados na Tabela 1 variaram de 5,14 a $7,72 \times 10^{-12} \mathrm{~m}^{2} / \mathrm{s}$ para os grãos de cevada, com diferença significativa entre os mesmos $(p \leq 0,05)$. De forma geral os valores apresentados pelos cultivares foram semelhantes. Observa-se que o coeficiente de difusão aumentou com o aumento da temperatura. $\mathrm{O}$ maior coeficiente de difusão foi na temperatura de $20^{\circ} \mathrm{C}$. A partir da temperatura de $25^{\circ} \mathrm{C}$ até $35^{\circ} \mathrm{C}$ houve diminuição desses valores. Nas temperaturas maiores no tempo de 32 horas os grãos se desintegraram porque já tinham atingido a umidade de equilíbrio a partir do tempo de 24 horas, aumentando a perda de sólidos, provavelmente por isso o coeficiente de difusão diminuiu nestas temperaturas, observando-se que a faixa de temperatura de 30 e $35^{\circ} \mathrm{C}$ é muito alta para o processo de hidratação da cevada devido à alta velocidade de hidratação inicial, os grãos tendem a fragmentar-se em poucas horas de hidratação. Segundo Prasad et al. (2010) o aumento da temperatura altera a taxa de difusão e afeta o comportamento da absorção. Um aumento excessivo da temperatura pode prejudicar a integridade da estrutura, promover a gelatinização do amido e afetar o comportamento de ganho de peso.

Tabela 1 - Difusividade efetiva da cevada nas temperaturas entre 10 e $35^{\circ} \mathrm{C}$.

\begin{tabular}{l|l|l}
\hline & $10^{\circ} \mathrm{C}$ & $5,14 \times 10^{-12}$ \\
& $15^{\circ} \mathrm{C}$ & $6,80 \times 10^{-12}$ \\
& $20^{\circ} \mathrm{C}$ & $7,72 \times 10^{-12}$ \\
& $25^{\circ} \mathrm{C}$ & $5,40 \times 10^{-12}$ \\
& $30^{\circ} \mathrm{C}$ & $6,42 \times 10^{-12}$ \\
& $35^{\circ} \mathrm{C}$ & $6,58 \times 10^{-12}$ \\
\hline
\end{tabular}




\section{9 a 22 de outubro de 2014 \\ Florianópolis/SC}

Estudos realizados por Mayolle et al. (2012) encontraram valores de coeficiente de difusão variando de 5,28 a $7,81 \times 10^{-12} \mathrm{~m}^{2} / \mathrm{s}$ para quatro cultivares de grãos de cevada inteiros e 35,2 a $49,5 \mathrm{x}$ $10^{-12} \mathrm{~m}^{2} / \mathrm{s}$ para o endosperma da cevada. Os dois experimentos foram realizados na temperatura de $22^{\circ} \mathrm{C}$. Tagawa et al. (2003) encontraram valores de 3,5 a $3,9 \times 10^{-12} \mathrm{~m}^{2} / \mathrm{s}$ também para cevada na faixa de temperatura de 10 a $50^{\circ} \mathrm{C}$. Neste artigo os valores do coeficiente de difusão foram superiores aos encontrados por Tagawa et al. (2003), e foram semelhantes aos estudos de Mayolle et al. (2012) para grãos inteiros. Em estudos realizados por Mayolle et al. (2012) com grãos de cevada inteiros e o endosperma da cevada, os autores constataram que a máxima absorção de água é de 5 a $10 \%$ menor nas amostras de endosperma comparada com os grãos inteiros sugerindo uma significante contribuição da casca e do gérmen na captação de água global.

Comparando o coeficiente de difusão da cevada com outros grãos, pode-se observar que o coeficiente de difusão é dependente das características físico-químicas e estruturais dos grãos estudados. Prasad et al. (2010) encontram valores de difusividade variando de 1,92 a $3,23 \times 10^{-9} \mathrm{~m}^{2} / \mathrm{s}$ para grãos de bico nas temperaturas de 40 a $60^{\circ} \mathrm{C}$. Resio et al. (2005) encontraram valores 0,27 a 3,70 x $10^{-11} \mathrm{~m}^{2} / \mathrm{s}$ para grãos de amaranto nas temperaturas de 30 a $90^{\circ} \mathrm{C}$. Khazaei \& Mohammadi (2009) encontraram valores de 4,16 a $6,97 \times 10^{-11} \mathrm{~m}^{2} / \mathrm{s}$ para grãos de gergelim nas temperaturas de 27 a 60 ${ }^{\circ} \mathrm{C}$. Os valores encontrados para cevada são superiores aos demais grãos, provavelmente devido sua a composição química e estrutura física.

\section{CONCLUSÕES}

No Cultivar BRS 195 a absorção de água é mais rápida nos estágios iniciais, principalmente nas primeiras oito primeiras horas e diminuiu gradualmente à medida que o conteúdo de umidade se aproxima da saturação. A hidratação da cevada é realizada em camadas e quanto maior a temperatura, maior é a velocidade de absorção de água no processo de hidratação. Os coeficientes difusão efetivos apresentados variaram de 5,14 a $10,8 \times 10^{-12} \mathrm{~m}^{2} / \mathrm{s}$. O coeficiente de difusão aumentou com o aumento da temperatura, o maior coeficiente de difusão foi na temperatura de $20^{\circ} \mathrm{C}$. A partir da temperatura de $25^{\circ} \mathrm{C}$ até $35^{\circ} \mathrm{C}$ ocorreu um aumento seguido de diminuição desses valores.

\section{REFERÊNCIAS}

ASSOCIATION OF OFFICIAL ANALYTICASL CHEMISTS (AOAC), Official methods of analysis of AOAC, 16 ed.; Arlington, v.2, 1995.

BARREIRO, J. A.; FERNANDEZB, S.; SANDOVAL, A. J. Water sorption characteristics of six rowbarley malt (Hordeum vulgare). Lebensm,-Wiss, U,-Technology, v. 36, p. 37-42, 2003.

BELLO, M.; TOLABA, M.; SUAREZ, C. Factors affecting water uptake of rice grain during soaking. Lebensm.-Wiss. u.-Technolgy, v. 37, n. 8, p. 811-816, 2004.

CRANK, J. The Mathematics of Diffusion. Oxford: Oxford University Press, Ely House, London Press, 1975. 
EMBRAPA. Empresa Brasileira de Pesquisa Agropecuária. Disponível em < http://www,cnpt,embrapa,br/culturas/cevada/cultivares/folder,pdf $>$. Acessado em 08/02/2014.

GEANKOPLIS, C.J. Transport Processes: Momentum, Heat and Mass (Allyn and Bacon series in engineering. 350p, 1983.

KASHANINEJAD, M.; MAGHSOUDLOU, Y.; RAFIEE, S.; KHOMEIRI, M. Study of hydration kinetics and density changes of rice (TaromMahali) during hydrothermal processing. Journal of Food Engineering, v. 79, n. 4, p.1383 -1390, 2007.

KHAZAEI, J.; MOHAMMADI, N. Effect of temperature on hydration kinetics of sesame seeds (Sesamumindicum L.). Journal of Food Engineering, v. 91, n. 4, p. 542-552, 2009.

MAYOLlE A. J. E.; LULLIEN-PELLERIN. V.; CORBINEAU B. F.; BOIVIN. P.; GUILLARD. V. Water diffusion and enzyme activities during malting of barley grains: A relationship assessment. Journal of Food Engineering, v. 109, p. 358-365; 2012.

PRASAD, K.; VAIRAGAR, P. R.; BERA, M. B. Temperature dependent hydration kinetics of Cicerarietinum splits. Food Research International, v. 43, n. 2, p. 483-488, 2010.

RESIO, A. C.; AGUERRE, R. J.; SUAREZ, C. Hydration kinetics of amaranth grain. Journal of Food Engineering, v.72, n. 3, p.247-253, 2006.

SHARMA, P.; GUJRAL, H. S. \& ROSELL, C. M. Effects of roasting on barley b-glucan, thermal, textural and pasting properties. Journal of Cereal Science, v.53, n. 1, p. 25-30, 2011.

TAGAWA, A.; MURAMATSU, Y.; NAGASUNA, T.; YANO, A.; IIMOTO, M.; MURATA, $\mathrm{S}$. Water absorption characteristics of wheat and barley during soaking. Transactions of the ASAE $\mathrm{v}$. 46, n. 2, p.361-366, 2003.

ULLRICH. S.E.; BAIK. B. Barley for food: Characteristics. improvement and renewed interest. Journal of Cereal Science, v. 48, p. 233-242, 2008. 